\title{
A CLASS OF INTEGRAL EQUATIONS INVOLVING LAGUERRE POLYNOMIALS AS KERNEL
}

\author{
by K. N. SRIVASTAVA \\ (Received 15th July 1964)
}

\section{Introduction}

Recently Widder (3) has obtained inversion integrals for a convolution transform whose kernel is a Laguerre polynomial. Buschman (1) has considered convolution equations with generalised Laguerre polynomial kernel. His result includes that of Widder. However no attempt has so far been made towards the study of singular integral equations involving Laguerre polynomials as kernel. Here the author obtains an inversion integral for such an equation.

The operator $\mathscr{F}_{n}$ and its properties

The operator $\mathscr{F}_{n}$ occurring in this note is defined by the formula

$$
\mathscr{F}_{n}\{f(x)\}=\frac{e^{-x}}{\Gamma(n)} \int_{x}^{1}(y-x)^{n-1} e^{y} f(y) d y .
$$

If $n$ is a positive integer, then by explicit computation we have

$$
\begin{gathered}
\frac{d^{n}}{d x^{n}}\left[e^{x} \mathscr{F}_{n}\{f(v)\}\right]=(-)^{n} e^{x} f(x), \\
\mathscr{F}_{n}\{f(x)\}=0 \text { for } x=1 .
\end{gathered}
$$

Integral equations and their solutions

Consider the integral equations

$$
\int_{\sigma}^{1} \frac{L_{n}^{-\alpha}(u-\sigma) y(u)}{(u-\sigma)^{\alpha}} d u=f(\sigma), \sigma \in I, n=1,2,3,4, \ldots
$$

where the integral is taken in the Riemann sense, $I=\{\sigma: c \leqq \sigma \leqq 1\}, c>0$ is a constant and $f(\sigma)$ is defined on $I$. It is assumed that

(a) $0<\alpha<1$,

(b) $f^{k}(1)=0$ for $0 \leqq k \leqq 2 n$,

(c) $f^{2 n+1}(\sigma)$ is piecewise continuous on $I$. If these conditions are satisfied, then the solution of (4) is given by

$$
y(u)=-A(n, \alpha) \int_{u}^{1} \frac{L_{n}^{\alpha-1}(v-u)}{(v-u)^{1-\alpha}} \mathscr{F}_{2 n}\{F(v)\} d v
$$

E.M.S. $-\mathrm{C}$ 
where

$$
\begin{aligned}
A(n, \alpha) & =\{(n) !\}^{2} / \Gamma(n-\alpha+1) \Gamma(n+\alpha), \\
F(v) & =\frac{d^{2 n+1}}{d v^{2 n+1}}\{f(v)\}
\end{aligned}
$$

and $L_{n}^{-\alpha}(x), n=1,2,3, \ldots$ are Laguerre polynomials.

As a preparation for the proof of the dual relation (4) and (5) we ha ve to establish the following summation result. It can be easily shown that for $n \geqq 1$

$$
\sum_{m=0}^{n} \sum_{r=0}^{n} \frac{z^{m+r}}{(n-m) !(n-r) !(m+r) !(r) !(m) !}=\{(n) !\}^{2} L_{2 n}(-z) .
$$

To prove this, we consider the sum

$$
S=\sum_{m=0}^{n} \sum_{r=0}^{n} a_{m, r} z^{m+r}=\sum_{p=0}^{2 n} c_{p} z^{p}
$$

where

and

$$
a_{m, r}=[(n-m) !(n-r) !(m+r) !(r) !(m) !]^{-1},
$$

$$
\begin{aligned}
c_{p} & =\sum_{s=0}^{p} a_{s, p-s} \quad \text { for } p \leqq n ; \\
& =\sum_{s=0}^{2 n-p} a_{s+p-n, n-s-1} \text { for } p \geqq n+1 .
\end{aligned}
$$

Substituting the value of $a_{m, r}$, we get

Hence our result follows.

$$
c_{p}=\left(\begin{array}{c}
2 n \\
p
\end{array}\right)\{(p) !\}^{-1}\{(n) !\}^{-2} \text {. }
$$

\section{Proof of the dual relation}

By virtue of the conditions $(a),(b)$ and $(c)$ it is obvious that the integral (5) exists and that the double integral

$$
J=-A(n, \alpha) \int_{\sigma}^{1} \frac{L_{n}^{-\alpha}(u-\sigma)}{(u-\sigma)^{\alpha}}\left(\int_{u}^{1} \frac{L_{n}^{\alpha-1}(v-u)}{(v-u)^{1-\alpha}} \mathscr{F}_{2 n}\{F(v)\} d v\right) d u .
$$

obtained by directly substituting (5) in the left-hand side of (4) is convergent. This double integral can be written as

$$
J=-A(n, \alpha) \int_{\sigma+\varepsilon}^{1-\varepsilon} \int_{u+\varepsilon}^{1} \frac{L_{n}^{-\alpha}(u-\sigma) L_{n}^{\alpha-1}(v-u)}{(u-\sigma)^{\alpha}(v-u)^{1-\alpha}} \mathscr{F}_{2 n}\{F(v)\} d v d u
$$

Since the integrals are uniformly bounded and there are at most a finite number of discontinuities of $F(v)$ in the region

$$
R:\left\{\begin{array}{l}
u+\varepsilon \leqq v \leqq 1 \\
\sigma+\varepsilon \leqq u \leqq 1
\end{array}\right.
$$


it is justifiable to interchange the order of integration in $R$. Thus we obtain

$$
J=-\underset{\lim \varepsilon \rightarrow 0}{A(n, \alpha)} \int_{\sigma+2 \varepsilon}^{1} \mathscr{F}_{2 n}\{F(v)\} d v \int_{\sigma+\varepsilon}^{\sigma-\varepsilon} \frac{L_{n}^{-\alpha}(u-\sigma) L_{n}^{\alpha-1}(v-u)}{(u-\sigma)^{\alpha}(v-u)^{1-\alpha}} d u .
$$

Because $L_{n}^{-\alpha}(u-\sigma) L_{n}^{\alpha-1}(v-u)$ is continuous and finite in the interval $v \leqq u \leqq \sigma$ and

the integral

$$
\int_{0}^{v}(u-\sigma)^{\alpha}(v-u)^{1-\alpha} d u=\pi / \sin \pi \alpha,
$$

$$
\int_{\sigma}^{v} \frac{L_{n}^{-\alpha}(u-\sigma) L_{n}^{\alpha-1}(v-u)}{(u-\sigma)^{\alpha}(v-u)^{1-\alpha}} d u
$$

exists, so that we can write

$$
J=-A(n, \alpha) \int_{\sigma}^{1} \mathscr{F}_{2 n}\{F(v)\} d v \int_{\sigma}^{v} \frac{L_{n}^{-\alpha}(u-\sigma) L_{n}^{\alpha-1}(v-u)}{(u-\sigma)^{\alpha}(v-u)^{1-\alpha}} d u .
$$

From ((2), p. $188(7))$ we have

Hence

$$
L_{n}^{\alpha}(x)=\sum_{m=0}^{n}\left(\begin{array}{c}
n+\alpha \\
n-m
\end{array}\right) \frac{(-x)^{m}}{(m) !} .
$$

$L_{n}^{-\alpha}(u-\sigma) L_{n}^{\alpha-1}(v-u)=\sum_{m=0}^{n} \sum_{r=0}^{n} \frac{\Gamma(n-\alpha+1) \Gamma(n+\alpha)(-)^{m+r}(u-\sigma)^{m}(v-u)^{r}}{(n-m) !(n-r) ! \Gamma(m-\alpha+1) \Gamma(\alpha+r)(r) !(m) !}$.

Substituting (11) in (10), writing $u-\sigma=x(v-\sigma)$ and making use of the following Euler's integral of the first kind

$$
\int_{\sigma}^{v}(u-\sigma)^{m-\alpha}(v-u)^{\alpha+r-1} d u=(v-\sigma)^{m+r} \frac{\Gamma(m-\alpha+1) \Gamma(\alpha+r)}{(m+r) !},
$$

(10) reduces to

where

$$
J=-\{(n) !\}^{2} \int_{\sigma}^{1} \mathscr{F}_{2 n}\{F(v)\} B(v-\sigma) d v
$$

$$
B(v-\sigma)=\sum_{m=0}^{n} \sum_{r=0}^{n} \frac{(\sigma-v)^{m+r}}{(n-m) !(n-r) !(m+r) !(r) !(n) !} .
$$

By (6), we have after using Rodrigues' formula for the Laguerre polynomial

$$
\begin{aligned}
B(v-\sigma) & =L_{2 n}(v-\sigma) /\{(n) !\}^{2} \\
& =\frac{e^{v}}{(2 n) !\{(n) !\}^{2}} \frac{d^{2 n}}{d v^{2 n}}\left\{e^{-v}(v-\sigma)^{2 n}\right\} .
\end{aligned}
$$

Introducing (14) in (13), we get

$$
J=-\frac{1}{(2 n) !} \int_{\sigma}^{1} \frac{d^{2 n}}{d v^{2 n}}\left\{e^{-v}(v-\sigma)^{2 n}\right\}\left[e^{v} \mathscr{F}_{2 n}\{F(v)\}\right] d v .
$$


Successive integrations by parts and application of the properties (2) and (3) give

$$
J=-\frac{1}{(2 n) !} \int_{\sigma}^{1}(v-\sigma)^{2 n} \frac{d^{2 n+1}}{d v^{2 n+1}}\{f(v)\} d v .
$$

Further successive integrations by parts and application of the conditions $f^{k}(1)=0,0 \leqq k \leqq 2 n$, finally yield

$$
J=f(\sigma)
$$

I am thankful to the referee for his valuable suggestions.

\section{REFERENCES}

(1) R. G. Buschman, Convolution equations with generalised Laguerre polynomial kernels, Notices Amer. Math. Soc., 10 (4) (1963), 374.

(2) A. ErdelyI, Higher Transcendental Functions, Vol. 2 (McGraw-Hill, New York, 1953).

(3) D. V. WIDDER, The inversion of a convolution transform whose kernel is a Laguerre polynomial, Amer. Math. Monthly, 70 (1963), 291-293.

M.A. College of Technology

Bhopal (M.P.), INDIA 